\title{
Los barrios de origen industrial ante el desafío de la desindustrialización. El "ponent" de Tarragona, entre la marginalidad y el anhelo urbano.
}

\author{
Juan Manuel Zaguirre Fernández $\mathbf{1}$, Josep Maria Solé Gras² \\ Centre de Recerca Urbana del Camp (CRUC). Unidad Predepartamental de Arquitectura. Escuela \\ Técnica Superior de Arquitectura. Universitat Rovira i Virgili. Reus, Tarragona, España \\ E-mail: 1juanmanuel.zaguirre@urv.cat, 2josepmaria.sole@urv.cat
}

\begin{abstract}
Resumen. El interés de esta propuesta de comunicación radica en revelar los desafios entre la Tarragona (Cataluña) compacta de límites históricos, reconocibles y consolidados y sus barrios dispersos de origen industrial. Sobre terrenos cuarterados "entrevías", y apoyándose sobre la nueva geometría impuesta por las lógicas de la infraestructura de la movilidad, se desplegó una condición periférica que la ciudad ignoró y de la que rápidamente se apropiaron las fuerzas productivas. Hoy, ante el desafio de la desindustrialización y sus derivadas urbanas en estos barrios de origen industrial, la investigación aborda tanto los efectos de la renuncia de la ciudad de Tarragona a incorporar esta periferia en términos de vulnerabilidad y segregación derivada como la dualidad generada con ello en términos de paisaje resultante. Bajo esta perspectiva, y con el fin de averiguar y comprender esta anomalía, deducimos que, para el caso de Tarragona, existió -y existe todavía- un "orden inverso", es decir, que la construcción de la periferia emana desde el territorio hacia la ciudad y no de la ciudad al territorio, motivando un desarrollo en paralelo de estas dos dinámicas. El resultado ha sido la emergencia de un "Distrito Distinto" que está rebasando las expectativas industriales y que de su correcto manejo depende la solución de esta controversia. En este sentido, la parte final de esta investigación explora posibles lineas estratégicas de transformación de unos suelos de alto valor urbano, productivo, social y de paisaje cuya imagen está por redibujar.
\end{abstract}

Palabras clave: Tarragona, Barris de Ponent, industrialización marginal, periferia, desindustrialización

\section{Motivación y antecedentes}

Este trabajo trata de reconocer los procesos de ocupación y la escenografía compleja y oculta sobre la que se ha producido la construcción de la periferia industrial y desarrollista de Tarragona en un entorno concreto como es el polígono industrial de Entrevías. Como su propia toponimia anticipa, apoyándose sobre una nueva geometría derivada de la acumulación continuada de trazados impuestos desde la lógica de la movilidad, sobre este lugar se desplegó una condición periférica que la ciudad ignoró de manera permanente cediendo, con ello, su control a las nuevas fuerzas productivas que impusieron su relato urbano. Ante esta circunstancia, la investigación - de la que se deriva esta comunicación- aborda los efectos de la renuncia de la ciudad de Tarragona a incorporar esta periferia y de la dualidad que esto ha provocado. Tradicionalmente, las descripciones de este emplazamiento han basculado entre la determinación de las infraestructuras y el magnetismo de la estricta zonificación residencial e industrial. De este modo, mientras que las primeras han

http://dx.doi.org/10.4995/ISUFh2019.2019.9660

(c) EY-NC-ND 2019, Editorial Universitat Politècnica de València 
procurado una segmentación territorial en el tiempo que ha beneficiado la confección del tejido fraccionado que ha llegado hasta nuestros días, las segundas han protegido la especificidad de cada una de ellas preservando una distancia suficiente capaz de neutralizar conflictos, diluirlos y disponer del necesario margen de maniobra para que la vida urbana ${ }^{1}$, en múltiples formas, arraigara precisamente en los lugares intersticiales y de fricción entre conflictos y contradicciones.

\section{Iniciativa y objetivos}

La iniciativa obedece a un interés en reivindicar, desde el manejo del análisis de la forma y de sus estructuras, la oportunidad que ofrece la estimulación urbana de los trazados infraestructurales (vías) y de sus huecos "intraestructurales" (entrevías²), de explorar el gran potencial estratégico y escenarios de nuevas posibilidades urbanas que permitirían equilibrar, en el caso de Tarragona, el largo y tenso pulso entre ciudad y periferia.

La maduración viene dada, en gran medida, por el acarreo de materiales y conocimientos acopiados durante una reciente etapa predoctoral que ha permitido identificar los periodos y comprender los distintos instantes $\mathrm{y}$ voluntades por los que se forja una forma general en la periferia entrevías de la ciudad de Tarragona. Con ello, se aspira a contribuir en el aporte de un enfoque alternativo que, desde una orientación posibilista, plantee una organización urbana asequible basada en trazados $\mathrm{y}$ asentamientos cautivos por un estereotipo periférico-industrial en el que también emerja, silenciosamente, una identidad estructurada desde la desarticulación urbanística y estimulada por la fertilidad de la riqueza formal que proporciona esta situación entrevías. Se trata, pues, de identificar la especificidad de una particular condición periférica que, para el caso de Tarragona, revela un "orden inverso" en la construcción de la periferia que emana desde el territorio hacia la ciudad y no de la ciudad al territorio motivando, así, un desarrollo en paralelo de estas dos dinámicas. El objetivo es averiguar, reconocer y comprender cómo se han incorporado, desplegado y aglomerado sobre este territorio -originariamente agrícola-, sus

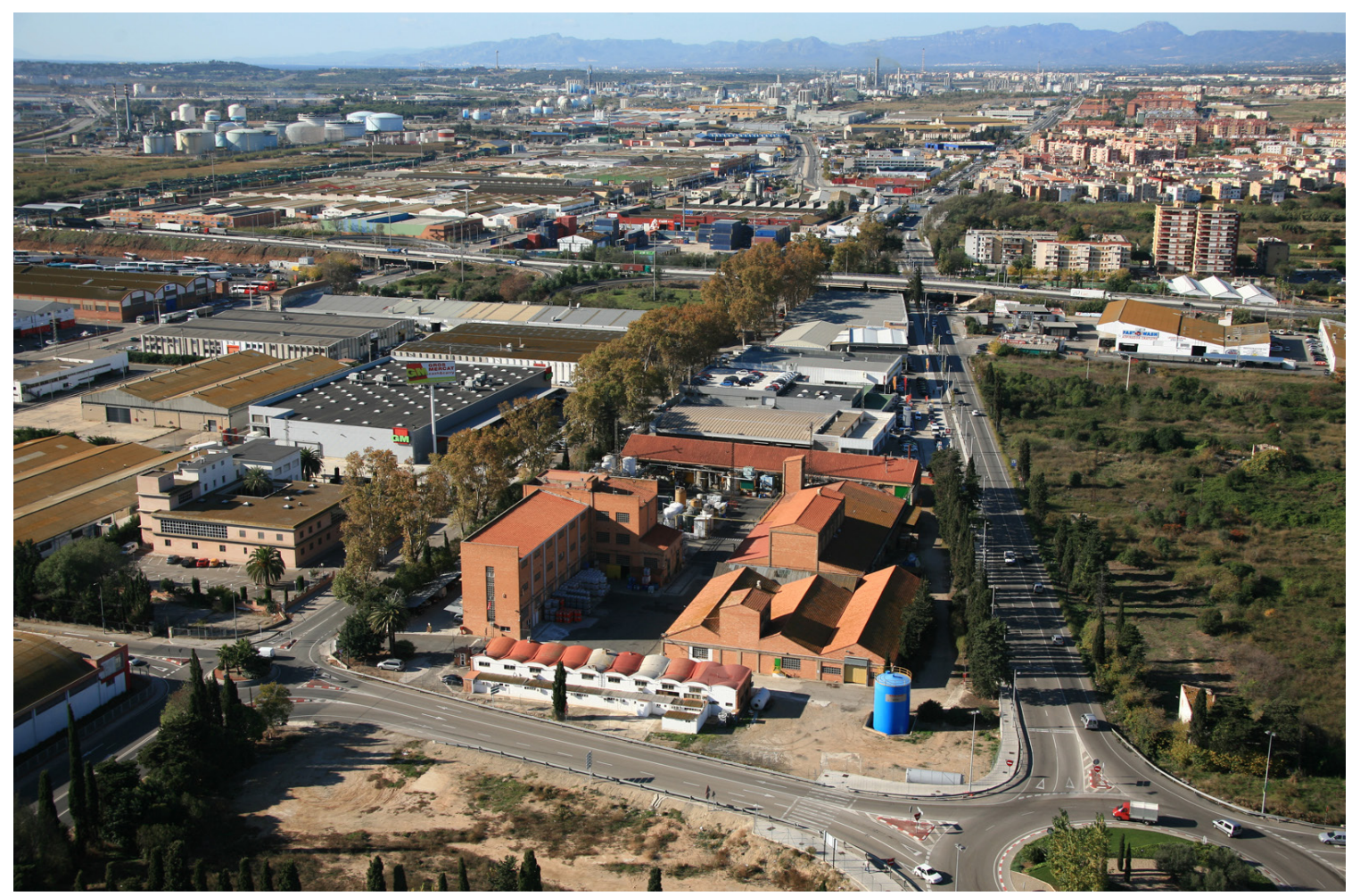

Fig. 1. Los barrios de Ponent de Tarragona y el polígono químico. Fuente: AVIOTEC, 2009 
nuevas estructuras socioeconómicas, dando lugar a un espacio fabricado por adición de usos cuyo resultado se manifiesta en la emergencia de un Distrito Distinto que está rebasando las expectativas industriales, desafiando el fenómeno de desindustrialización del monocultivo petroquímico y que, de su correcto manejo, depende una posible solución de esta controversia que no sería otra que el anhelo urbano de una periferia de origen industrial.

\section{Reacciones y ocupaciones espontáneas en la periferia de Tarragona}

Para Tarragona y otras tantas ciudades emergentes en el ocaso de la España autárquica de los cincuenta, la primera Ley del Suelo, de 1956, se convirtió en un buen instrumento para su transformación. En el caso tarraconense, con terrenos atrapados entre campos de cultivo, infraestructuras e industrias diseminadas, esta ley, aplicada sobre una sociedad desorientada por la trayectoria del progreso, legalizó la especulación que abrió el camino a la ocupación industrial de Entrevías. Las crónicas describen a una ciudad "estresada" por este fulminante y concentrado progreso. A partir de 1956, Tarragona recibió un gran empuje económico, sin parangón en el resto de España, para una provincia de economía débil y mentalidad estática.

\section{Parcelario, periferia y vivienda}

Después de veinticinco años de crisis política, social y económica, en Tarragona se encadenan crecimiento, necesidad y espontaneidad a partir de los años cincuenta cuando, entre los objetivos de política económica que apuntan hacia las formas más deseables del desarrollo económico y social, están los que se refieren al "reparto de la actividad económica en el espacio físico" (F. de Terán, 1973). De dicha voluntad deriva que el impulso de políticas económicas tenga un reflejo inmediato en el territorio, planificado o no. En pleno periodo autárquico, la materialización y localización de este reparto superó las históricas barreras que la ciudad había sostenido incluso con posterioridad al derribo de sus murallas. Siempre fueron unos límites más conceptuales que físicos, aunque, por primera vez, la ciudad tuvo en cuenta a su periferia en este reparto distribuyendo industria y vivienda en ámbitos discontinuos y alejados del núcleo urbano tradicional. De todos los asentamientos residenciales en el oeste de Tarragona, destacan los de Torreforta y Bonavista por su promiscuo nacimiento, por su rápida extensión y por su aparente estratégica ubicación con relación a toda traza infraestructural, quizás buscando el anonimato ocupando terrenos entrevías de escasa demanda o condicionados por la

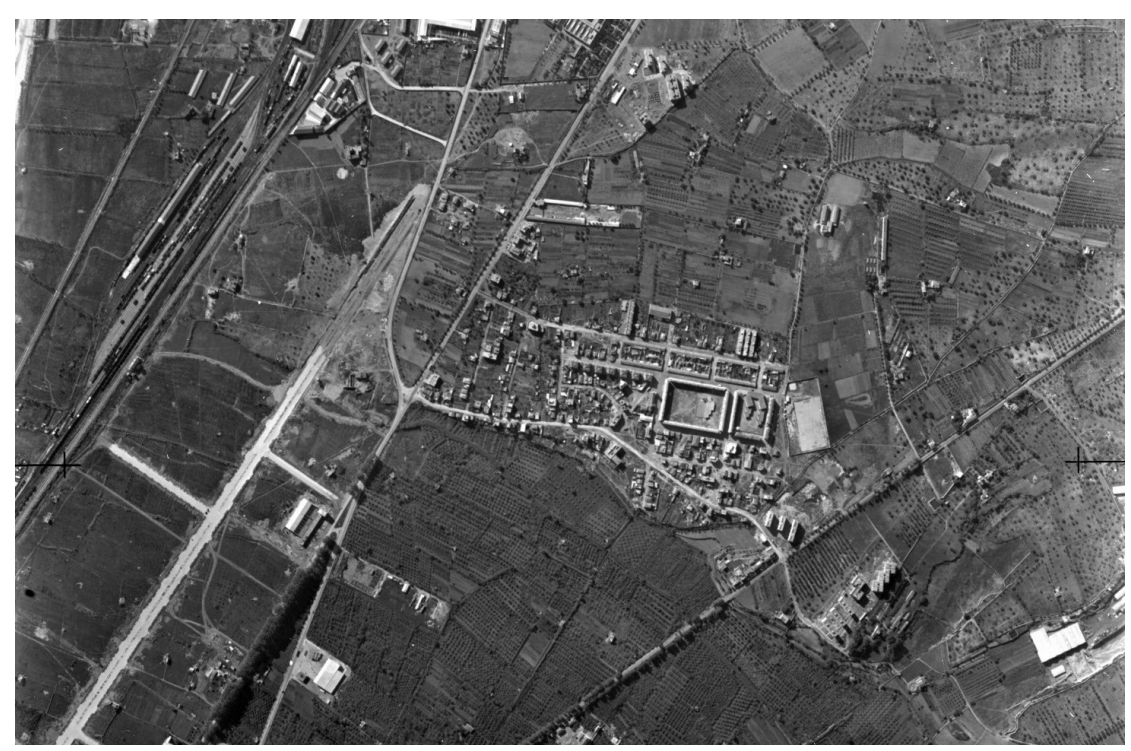

Fig. 2. La imagen de la alteración del parcelario. Industria y vivienda sobre el mosaico agrícola. Fuente: ICC, 1965 
búsqueda de terrenos de extensión y forma que los habilitase como parcelables. Es el plano parcelario elaborado a partir de los años treinta, denominado Catastrón ${ }^{3}$, el documento que mejor nos muestra el proceso de ocupación del territorio entrevías. Una imagen precisa de la estructura de la propiedad previa y mutante en función del proceso de colonización iniciado en los años cincuenta. Este documento, más allá de establecerse como principal tablero de trabajo y campo de pruebas de los proyectistas que empezaban a imaginar la periferia de Tarragona, también se acabó convirtiendo en el mayor instrumento de especulación del momento.

\section{Fábricas en el paisaje. La confirmación de una voluntad industrial, 1956-1959}

Para el desarrollo económico de Tarragona, el periodo entre 1956 y 1959 supone la confirmación de una voluntad industrial para la periferia oeste de la ciudad preservando, con ello, la zona este para el turismo nacional. En este sentido, las noticias de nuevas industrias interesadas en Entrevías acaparan la mayoría de las crónicas económicas del momento. Las nuevas y modernas condiciones de eficiencia logística y potencial de movilidad de bienes y materias primas (por ferrocarril, por medio de transporte marítimo y por carretera) sumado al acceso a la electricidad y una evidente capacidad de transformación atraen paulatinamente a industrias principalmente del sector petrolífero y químico, así como a pequeñas industrias auxiliares, de transformación, de alimentación y de automoción. Tal acumulación de circunstancias y parabienes tiene consecuencias económicas, sociales $y$, por supuesto, urbanísticas. En este periodo tan transcendental, la ciudad no tiene ni ideas ni planes, por lo que la imagen de la ciudad en este preciso momento responde precisamente a una improvisación urbanística que también ha participado en la construcción y consolidación de su forma urbis.

La mayor intuición que tenía la ciudad en 1956-1959 era el horizonte industrial que
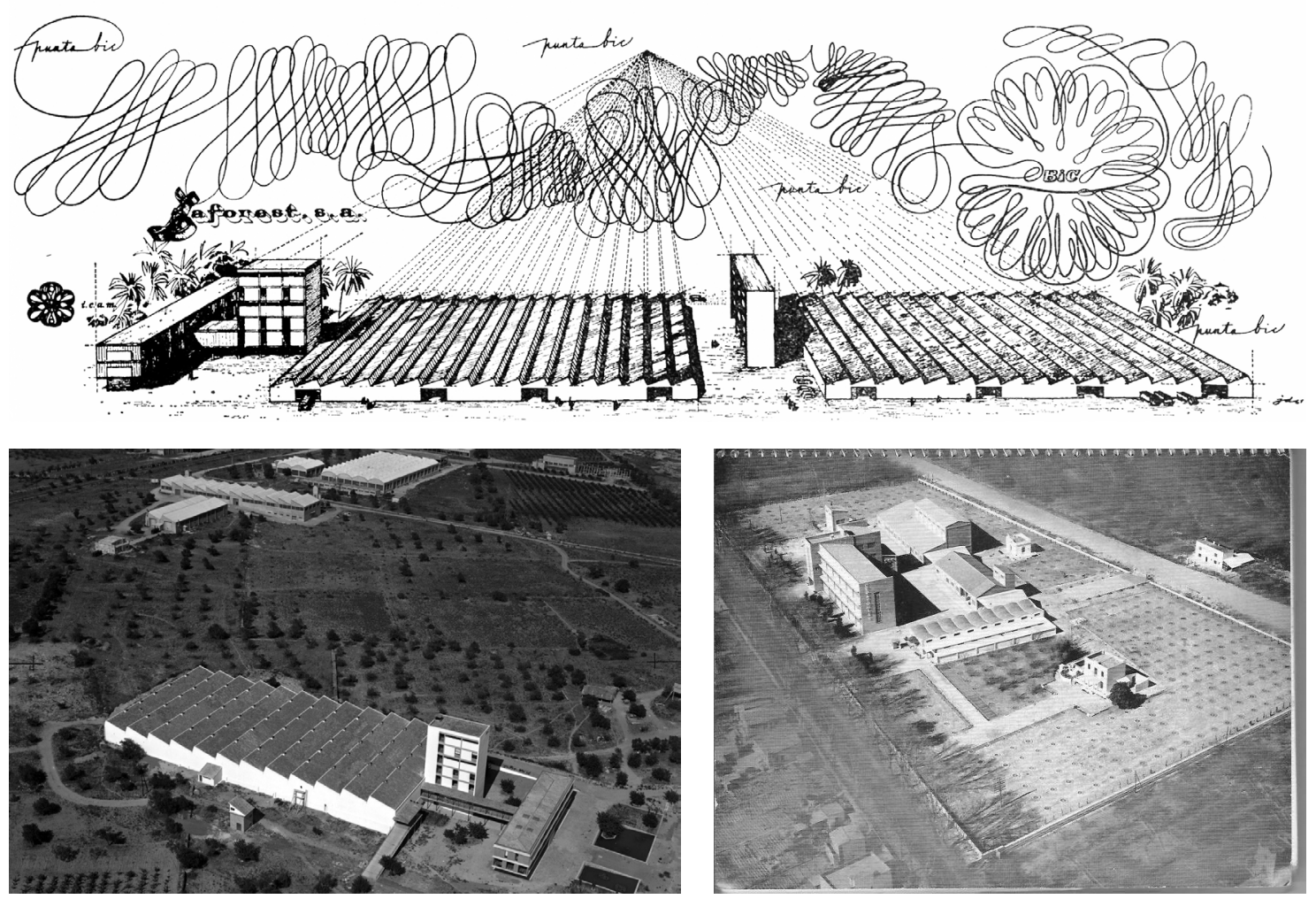

Fig. 3. Fábricas en el paisaje. Punta BIC (perspectiva y vista aérea), 1962 Fuente ICC. Ceratonia, 1962 Fuente: ATA 
Fig. 4. Imagen del proceso de ocupación industrial sujeto únicamente a las necesidades y características de producción y desvinculado de cualquier nexo o relación con tejido alguno. Fuente: ICC, 1966

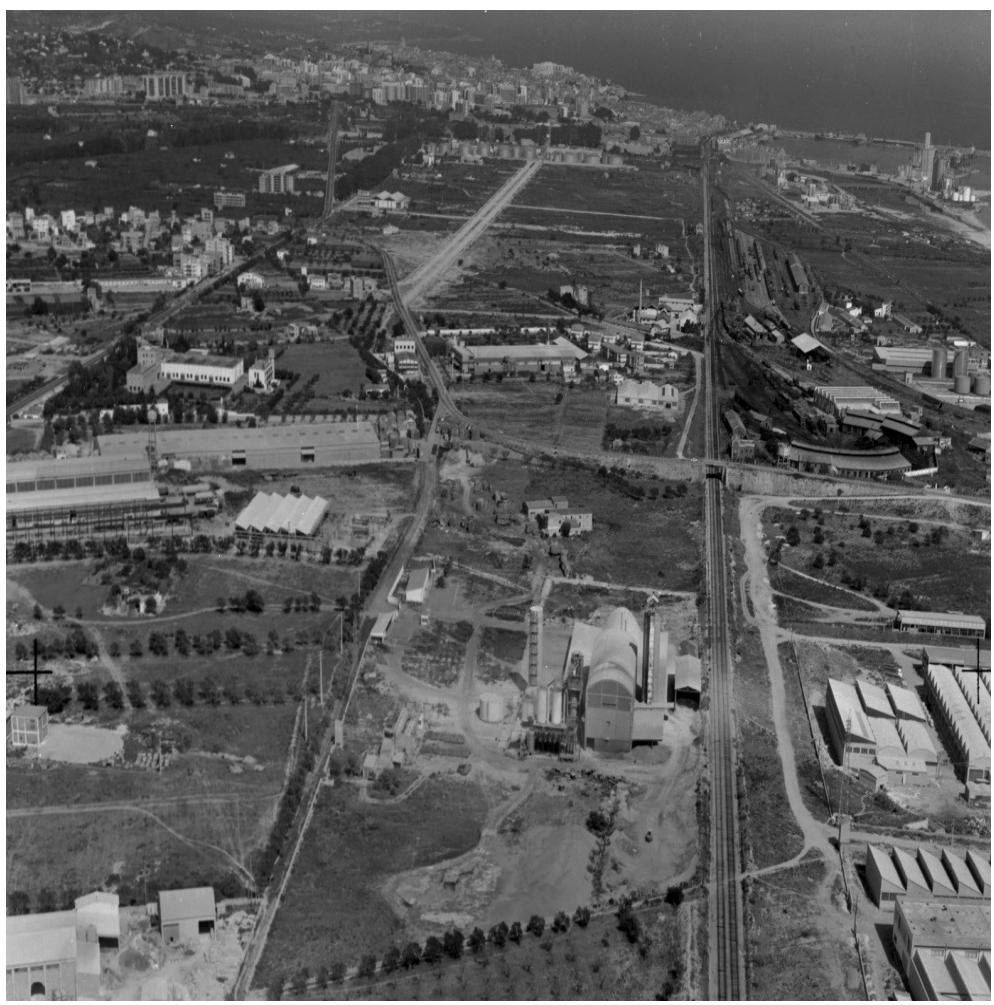

tenía por delante si acertaba en posicionarse como un polo económico complementario a la corona industrial de Barcelona que, dicho sea de paso, ya mostraba evidentes síntomas de saturación. Tarragona, atenta a esta deriva, supo aprovechar razonablemente su condición estratégica para consolidar una vocación industrial que ha perdurado durante décadas. El precio de esta intuición es sabido. Véase una gran extensión industrial que hizo suya la condición entrevías para evitar ser urbanizada a cualquier coste. Ante el evidente desajuste de este devenir, las tardías y escasas reacciones se materializaron en una mediocre urbanización del polígono industrial Entrevías y en el proceso de expropiación forzosa -gracias a la nueva Ley del Suelo- de terrenos destinados a la construcción del nuevo polígono industrial del Francolí (de impulso estatal). Entre tanto, la llegada de nuevas industrias por el efecto de concentración sectorial fue incesante, produciendo a la par otro efecto llamada, en este caso, de mano de obra barata ${ }^{4}$, que desbordó el precario y escaso parque de viviendas sociales de la ciudad alentando la parcelación espontánea del suelo agrícola próximo a las nuevas industrias en proyecto y construcción.

\section{La "industrialización marginal". Vinculaciones indisciplinadas en la periferia}

A raíz de la llegada de los nuevos fenómenos socioeconómicos ligados a la industrialización de Tarragona con un evidente impacto sobre el territorio, surgieron tejidos periurbanos de características marginales y no siempre vinculados al hecho residencial. Esta actividad económica e industrial, aceptada como fenómeno social de primer orden en la Tarragona moderna, se vio alterada a razón de una incorrecta implantación en el territorio al no reunir las condiciones de planificación, urbanización y edificación necesarias para un correcto funcionamiento. Con ello, se dio pie a que estos nuevos espacios de la ciudad, marcados por una intensa imagen industrial y con escasez urbanística no se convirtieran en espacios de precisión (R. Sennett, 1997) y por consiguiente generasen distancia y tensión en la imagen fabricada de la ciudad. Los momentos especulativos que marcaron la condición periférica industrial en la Tarragona moderna comienzan a transformar la estructura del suelo en Entrevías, dando lugar a establecimientos periurbanos -de marcadas características 
marginales- no vinculados a la presión residencial y que obedecen a una dinámica pro-industrial, a la precariedad urbanística y a la impunidad del momento. Este fenómeno de industrialización marginal ${ }^{5}$ vendría a ser el proceso de ocupación industrial intensiva, espontánea y dispersa identificado básicamente en la periferia. Reconocemos aquí un fenómeno de características similares a los asentamientos marginales residenciales que, por definición, han ocupado franjas de terreno destinadas al olvido y a la exclusión urbanística, invitando a abordar estos procesos de industrialización marginal en el territorio bajo ópticas de análisis, diagnosis e intervención próximas a esta forma de crecimiento urbano que se fundamenta en características estructurales de implantación marginal.

\section{"Distrito Distinto". El resultado de un orden inverso y simultáneo}

Una vez el proceso de ocupación descrito adquiere la suficiente fuerza y madurez, las diferentes figuras de planeamiento que elabora la ciudad de Tarragona a partir de 1960 señalan la potencia de este "Distrito distinto", pero, a la vez, esquivan el dibujo del proyecto para saltar el río Francolí (J. Parcerisa, 1991). La consecuencia fue que en terrenos entrevías $\mathrm{y}$, apoyándose sobre esta nueva geometría paralela a una ciudad que básicamente se ocupa de la racionalización de su centro y rápidamente reconocida por intereses económicos, se desplegó una condición periférica -por acumulación de trazados- que la ciudad ignoró y de la que las fuerzas productivas se apropiaron. Ante esta circunstancia, la investigación ha abordado los efectos de la renuncia de la ciudad de Tarragona a incorporar esta periferia y de la dualidad que esto ha provocado.

\section{EI Ponent de Tarragona ante el desafío de la desindustrialización}

Hoy, en un momento de incertidumbre territorial, el motor industrial químico y residencial periférico de Tarragona presenta un claro estancamiento y, en paralelo, evidentes tendencias a la "tercialización" del tradicional ámbito industrial al otro lado del Francolí. La escasa heterogeneidad industrial de Entrevías en la actualidad podría convertirse en un lastre en caso de una relajación productiva del hub químico, dejando inertes los espacios

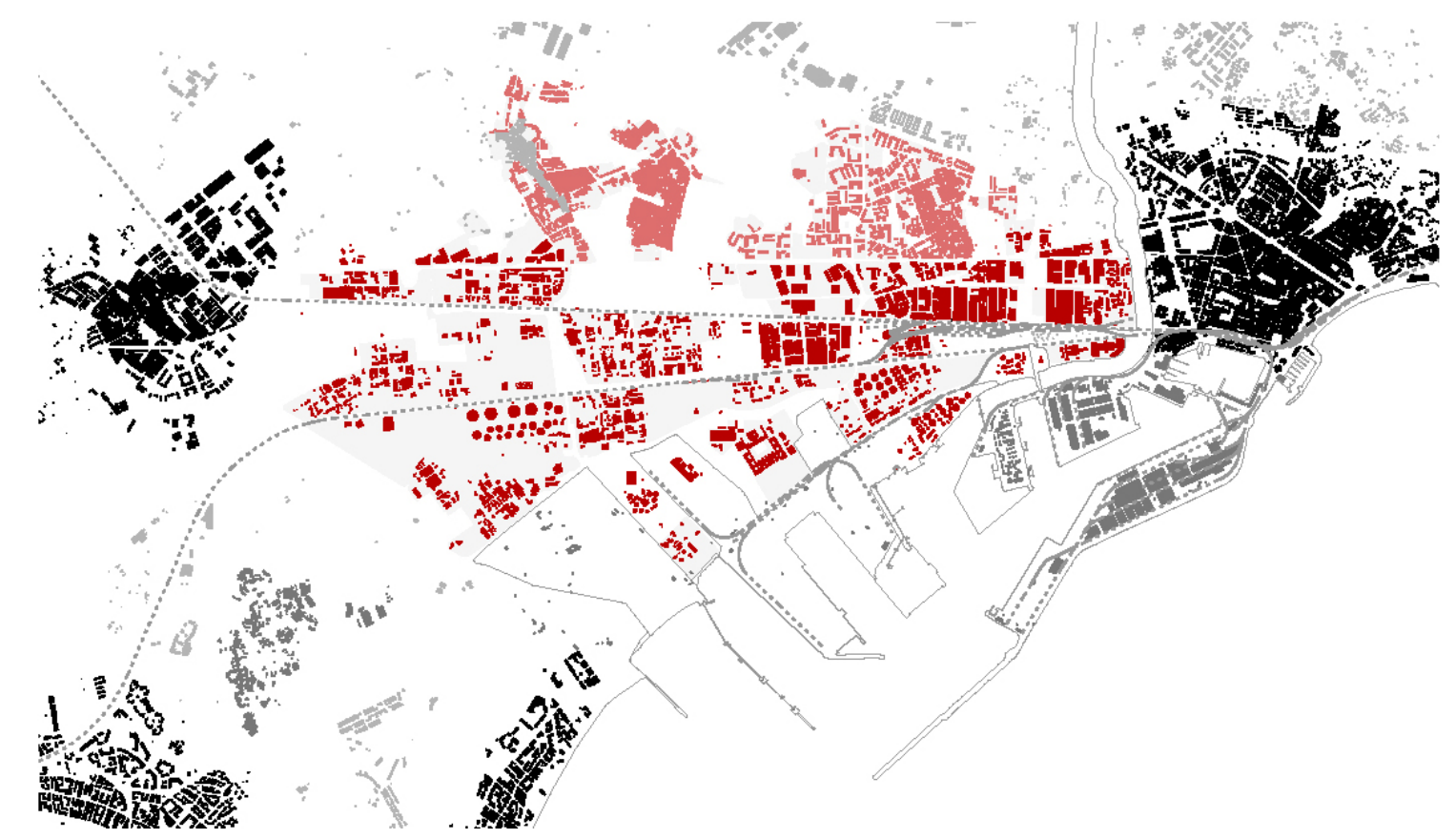

Fig. 5. La consolidación del distrito industrial definido por la N-340 y Entrevías confirma la administración de políticas bien diferenciadas que fortalecen la tesis de "una ciudad a dos velocidades" (Parcerisa, 1991). Fuente: Elaboración propia, 2018. 
intersticiales dispersos pensados para acoger pretéritas expectativas de ampliación. A esta renuncia de la ciudad por reconocer a su periferia como bien potencial en términos ecológicos o de paisaje se suma un proceso paralelo, lento pero implacable, de desindustrialización. No obstante, sigue vivo el anhelo urbano manifestado por la complejidad social y urbana de su condición, así como por la emergencia de nuevas centralidades, la oportunidad de sus tangencias y nodos de comunicación, sus privilegios industriales y por el beneficio de sus vacíos urbanos. Hoy, la dicotomía es más evidente que nunca pero, ¿hasta qué punto esta realidad difusa puede llegar a consolidarse más allá de una periferia de carácter preminentemente industrial?, ¿nos encontramos, entonces, ante la tesitura de forzar el ámbito de Entrevías a convertirse en un lugar del dominio de la racionalidad y el rigor urbanístico? $\mathrm{O}$, ¿se prefiere, en cambio, incorporar su talento urbano, paisajístico y territorial a la forma general de la ciudad asumiendo su origen peri-industrial y estimulando la fertilidad de su identidad desde su diversidad? Seguramente, este ejercicio pasaría por un delicado proceso de selección que sopesara los elementos de los que se puede prescindir y aquéllos que lo definen y de los que no debiera desprenderse. Como anticipaba Jane Jacobs, lo fácil que es caer en la trampa de considerar los usos de la ciudad cada uno por separado o por categorías ${ }^{6}$, es decir, simplificar en exceso la complejidad de la ecuación de una urbanidad difusa y de intensidades variables. No obstante, esta aproximación ha sido una práctica habitual al referirse al Ponent de Tarragona, donde ha prevalecido siempre la visión por categorías por encima del potencial de la diversidad de su tejido productivo y trasposición morfotipológica de sus usos. Puede que haya llegado el momento donde las circunstancias tan fundamentales para este territorio como el turismo, la industria, la residencia, las infraestructuras y lo rural sean observadas, analizadas, cuantificadas y planificadas con la voluntad e interés de combinarlos en soluciones de reequilibrio y escenarios de mezcla e hibridación.

Atendiendo a esta hipótesis $\mathrm{y}$, ante el reto de la progresiva desindustrialización que impera,

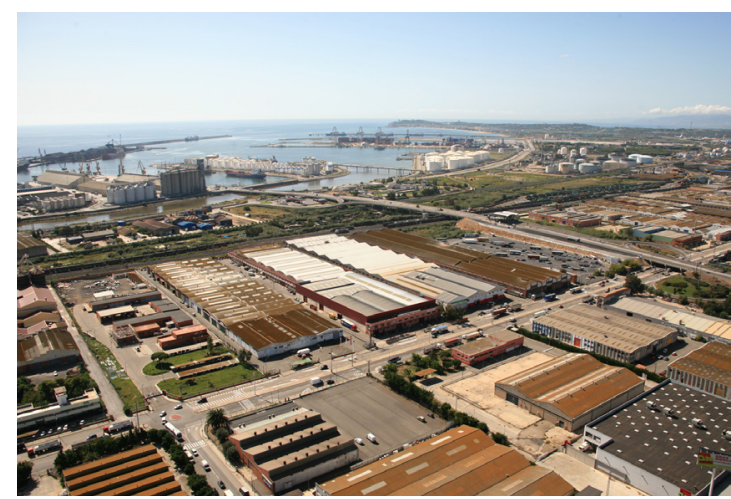

Fig. 6. Imagen aérea actual del Polígono Industrial del Francolí (1965), próximo a la trama urbana de la ciudad y en constante proceso de tercialización. Fuente: Aviotec, 2010.

la investigación toma en consideración una serie de escenarios como posibles candidatos a facilitar esta necesaria transición hacia la obligada incorporación del talento formal, paisajístico y arquitectónico de Entrevías de Tarragona a su Forma General de ciudad.

\section{El beneficio de los vacíos en el dibujo de la Gran Vía del Camp}

A lo largo de la investigación doctoral que da pie a esta comunicación se ha confirmado que, en el Camp de Tarragona, la dimensión lineal siempre cobra protagonismo. Por ello, su dibujo habría destapado expectativas insospechadas, llevando constantemente a la ciudad ante la tesitura de incorporar nuevos episodios en la construcción de su forma general a partir de la consolidación de ejes que incluso han llegado a desplazar en distintas ocasiones su centro de gravedad. Seguramente, de todos los elementos $\mathrm{y}$ circunstancias que participan en la imagen al pie, el conjunto de "vacíos" a lo largo de la $\mathrm{N}-340$ es el que atesora mayores posibilidades para activar este engranaje urbano y ayudar a resolver la dualidad que persiste entre ciudad y periferia. Una oportunidad que permanece latente gracias a las precauciones que, en forma de reservas, tomaron la industria, la residencia y las infraestructuras. Además, sobre este trazado interceptan la mayoría de atributos y circunstancias descritas en esta investigación: periferia, vacío, industria, marginalidad, huerta, paisaje e infraestructura. 


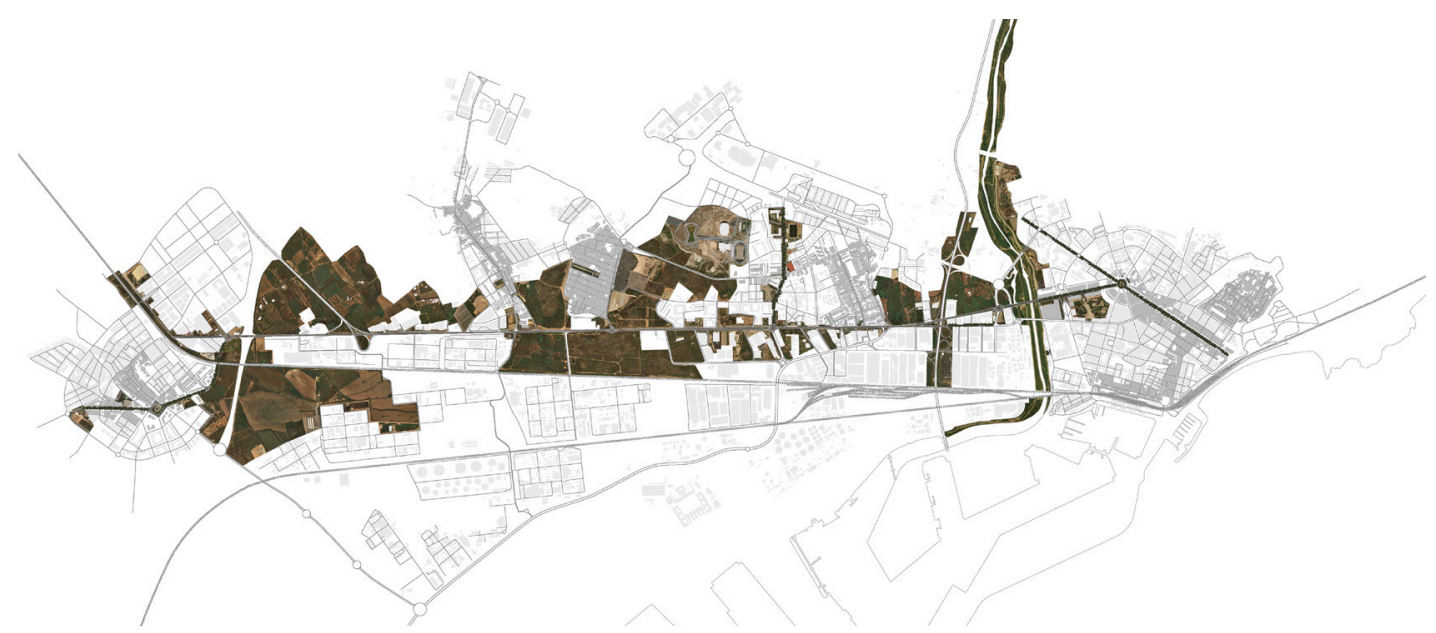

Fig. 7. El dibujo de la Gran via del Camp. Fuente: elaboración propia. 2018.

\section{La oportunidad de las tangencias. EI nodo intermodal de la estación Clasificación}

La especial acumulación de trazados y corredores estratégicos en el Camp que han dado lugar a una copiosa serie de tangencias $\mathrm{y}$ fricciones que apuntan una colección de situaciones territoriales que atesoran potenciales oportunidades de transformación hace, a nuestro juicio, demasiado comprometida la búsqueda de una única centralidad que sustituya los valores característicos de la estructura policéntrica de este territorio. En este sentido, probablemente, la mejor respuesta a esta creciente demanda de conectividad de un territorio fragmentado pasaría por el aprovechamiento de la intermodalidad latente que proporcionan muchas de estas tangencias. Mediante este enfoque, una de estas situaciones desvela un potencial estratégico por encima de otros. Se trata del ámbito de tangencia que dibujan los trazados actuales alrededor de la estación de clasificación de mercancías en Entrevías. Esta propuesta combinaría, con aparente mayor eficacia, los flujos de población que transporta la red de ferrocarril actualmente en servicio (corredor Móra-Reus-Tarragona y corredor Cambrils-Tarragona-Barcelona), junto a los del corredor del mediterráneo - una vez se asuma el inevitable bypass que recupere la circulación de trenes de largo recorrido por el centro de la ciudad-y las dinámicas propias de ubicar una estación en un entorno más urbano.

\section{Conclusiones}

Tradicionalmente, el Ponent de Tarragona ha basculado entre las tensiones ejercidas tanto por los vectores infraestructurales como por la presión residencial e industrial a las que se ha visto sometido un ámbito de fuertes transformaciones que han derivado en el dibujo de un territorio fragmentado. Sin embargo, esta estructura de usos discontinuos e intereses fragmentados conllevan la aparición de una serie de espacios intersticiales de medida suficiente y distancia apropiada para imaginar una posible concepción de escenarios de transformación que incorporen el proyecto de la complejidad como base para neutralizar los conflictos actuales, dirimir las contradicciones evidentes y capacitar la progresiva introducción tanto de la vida urbana como de los valores ecológicos y de paisaje inherentes a estos fragmentos territoriales.

De este modo, en la construcción de esta periferia de "orden inverso" -desde el territorio a la ciudad- el impulso industrial debe explorar fórmulas que incorporen soluciones residenciales que se sumen a aquellas de origen espontáneo e informal que hoy conforman los barrios de Ponent fabricando, con ello, un espacio constituido por adición de usos capaces de convivir. La pregunta, entonces, que parece imponerse es: ¿qué potencial recorrido tendría para los barrios residenciales el desafío de la desindustrialización que acecha al monocultivo 


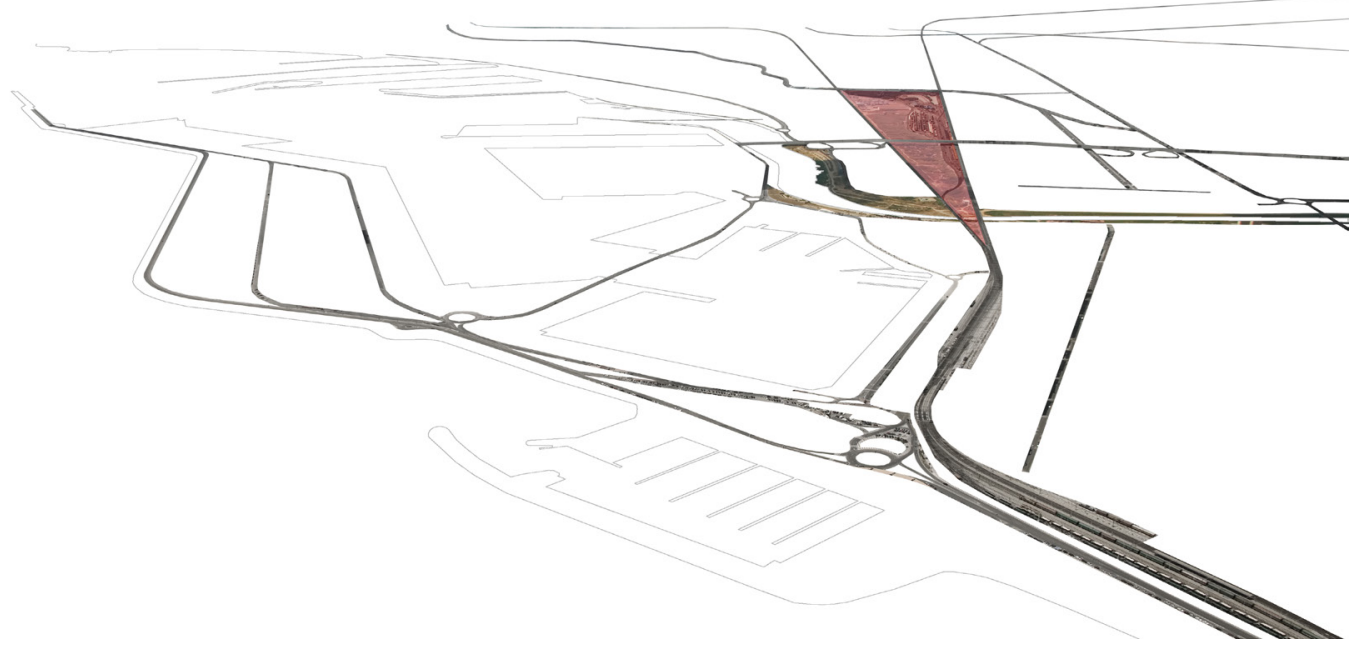

Fig. 8. La oportunidad de las tangencias. Sobre esta imagen despojada intencionadamente, sobre la cual se aprecia la interesante acumulación de infraestructuras en Entrevías, emerge una tangencia protagonizada por los terrenos de la estación Clasificación que permanece inalterada, y que reserva una oportunidad urbana aún por desvelar. Fuente: elaboración propia. 2018.

de la industria química en Entrevías?

Ante esta controversia y atendiendo a todo un arsenal de estímulos que propone el lugar, la investigación ha reflexionado hasta el momento sobre la paulatina digestión de fragmentos industriales en el umbral de la ciudad, un ámbito ya en transformación, salpicado de nuevos usos que ocasionan una interesante fricción que se podría zanjar de llevarse a cabo algunas operaciones de proyecto urbano candidatas a incorporarse a la Forma General de ciudad, ayudando así, a dibujar el necesario salto del río Francolí. Estas operaciones, sin duda, serían el proyecto de la Gran Vía del Camp y la Estación Intermodal de Ferrocarril en Clasificación. En este sentido, un estudio pormenorizado de ambas propuestas puede marcar las pautas de continuidad de esta línea de investigación iniciada.

Por último, otro de los posibles argumentos de investigación futuros que se dibujan pasaría por la mirada comparada, pautada y sistemática, de otros casos de estudio del que se pudieran extraer, pese a la evidente especificidad de cada uno de estos lugares, algunas posibles lecciones compartidas. Para ello, establecer metodologías que permitan este ensayo comparativo a partir de indicadores consistentes a lo largo del tiempo puede priorizarse como objetivo venidero.

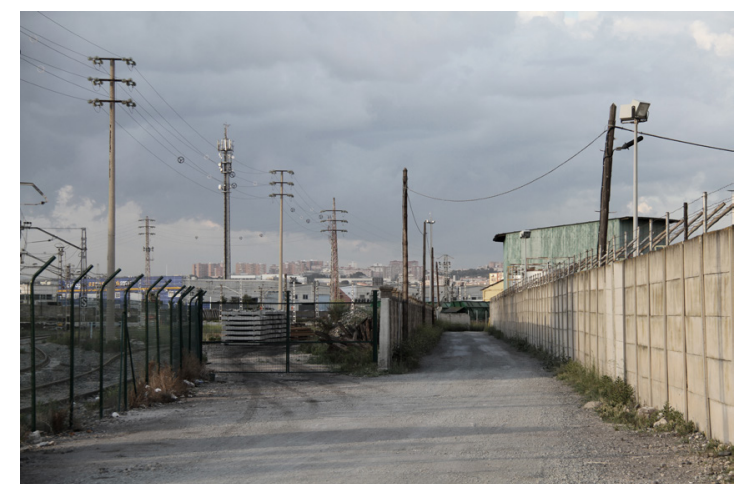

Fig. 9. Entrevías, un espacio latente.

Fuente: elaboración propia. 2019.

\section{Notas}

1 La imagen actual de la vida urbana del distrito industrial en la periferia entrevías de Tarragona, incorpora diversos rasgos claramente perceptibles y puestos de manifiesto en esta comunicación, de acuerdo con la amplia perspectiva que en ella se refleja y que se deriva de la tesis doctoral Vías y Entrevías. Léase: la cuestión industrial desde la óptica periférica y de amortización de territorios residuales, la evidencia articuladora de importantes ejes de comunicación como "polos" que atraen actividad y que, a la vez, actúan de límites y fronteras, el papel 
de las barreras físicas y geográficas en la expansión de las ciudades, y la mixticidad de usos y actividades que alberga un territorio dotado de grandes posibilidades.

2 Entrevías ha pasado en pocos años de ser un territorio agrícola y marginalizado por infraestructuras pasantes a ser motor esencial del desarrollo económico de la Tarragona moderna. Dotada prematuramente de atributos de ciudad, como centralidades, mercados tradicionales, ensanches, polígonos, equipamientos, monumentalidades, nodos, periferia e infraestructuras diversas, ha adquirido un compromiso urbano que le confiere cierta autonomía en lo económico, sobre todo en lo social, pero también en lo paisajístico. Este reconocido peso estratégico aportado por Entrevías no se traduce en una percepción de poseer cierta entidad urbanística -todo lo contrario- la precariedad y marginalidad periférica, ligada al hecho industrial, han sido su apelativo más habitual. ¿Pero, hasta qué punto esta realidad difusa puede llegar a consolidarse más allá de lo periférico?

3 Depositada en el Archivo Histórico de Tarragona se encuentra una serie de planimetría catastral producida a escala 1:2.000, con información topográfica $\mathrm{y}$ parcelaria, confeccionada a mano y que contiene diversas anotaciones manuscritas (algunas en color) añadidas y superpuestas en el transcurso de los años de vigencia del documento. Esta planimetría fue elaborada entre el intervalo 1932-1942 por la Brigada Topográfica de Parcelación de la Provincia de Tarragona, del Servicio de Catastro Topográfico Parcelario adscrito al Instituto Geográfico y Catastral, y posteriormente modificada, ampliada y revisada hasta disponer de una nueva planimetría elaborada sobre material fotográfico más actualizado -a partir de 1983- por empresas como OTECAR y EQUIPO CARTOGRÁFICO S.A.

4 "Queríamos mano de obra y llegaron personas." Max Frisch (arquitecto y escritor suizo), 1965.

5 Esta comunicación se alimenta de la investigación Vías y Entrevías (J. M. Zaguirre, J. Parcerisa. 2018) que reflexiona sobre cómo una ocupación industrial indisciplinada descubre las mejores condiciones de impunidad y aislamiento en los territorios entrevías que surgen de la acumulación de infraestructuras pasantes, dando lugar a una forma de ocupación con grandes similitudes con el crecimiento marginal.

6 Jane Jacobs. Muerte y vida de las grandes ciudades. Las condiciones para la diversidad urbana. Los generadores de diversidad. (pág. 172-210). Capitán Swing Libros. 2013

\section{Referencias}

Baldrich, Manuel. 1952. "La Ciudad-Comarca. Ante el crecimiento desmesurado de los aglomerados urbanos". Madrid: Boletín de información de la Dirección General de Arquitectura. Cuarto trimestre (VI): $19 \mathrm{p}$.

Bardají Ruiz, F. 2015. Bonavista. Una biografía social. Tarragona: Editorial Silva.

Del Amo, R. 2003. Transformaciones económicas y cambios sociodemográficos en el espacio de la conurbación TarragonaReus (1960-1996). Tesis Doctoral. Director: S. Roquer. Tarragona: URV.

De Terán, Francisco. 1973. "Algunos aspectos de las relaciones entre planificación física y planificación económica en la experiencia española". Madrid: DUyOT.ETSAM.UPM. Revista Ciudad y Territorio, num. 2, pág. 7-38.

Gómez Ordóñez, José Luis. 1982. El urbanismo de las obras públicas. Barcelona: Tesis Doctoral, LUB-UPC. Director de tesis, M. Solà-Morales.

Heynen, Hilde. 1992 "The Peripherial Condition. Some thoughts about a problematic phenomenon, illustrated by case study in Winterslag". Barcelona: Revista UR 9-10: 57-59 p. Proyectar la periferia, Laboratori d'Urbanisme ETSAB-UPC.

Jacobs, Jane. 1961. Muerte y vida de las grandes ciudades. Madrid. Capitan Swing Libros. Traducción 2011

Llovell Fortuny, F. 1980. La industrialización de Tarragona. Tarragona: Associació Empresarial Química de Tarragona (AEQT). 
Margalef, Joaquim. 1979. El Tarragonès, estructura econòmica: expansió industrial i desequilibris sectorials. Barcelona: Caixa de Catalunya ed.

Montejano, J. Antonio. 2010. Metropolización del Territorio y Regiones Urbanas Medias. El caso del ámbito central del Camp de Tarragona, 1977-2008. Tesis Doctoral. Director: Antonio Font. Barcelona: ETSABUPC.

Parcerisa Bundó, Josep. 1991. La forma urbis. Cinc ciutats sota sospita. Barcelona: Tesis Doctoral, UPC. Director de Tesis, M. SolàMorales.

Parcerisa Bundó, Josep. 2012. "Tarragona. Fundación y declinaciones de una forma general". En Forma Urbis. Cinco ciudades bajo sospecha. Barcelona: LUB.

Segarra, Agustí., Margalef, J., Teruel, M., Duro, J. A., Piñol, J. M., Miravet, D. 2007. El Camp de Tarragona: realitat actual i propostes per a la planificació estratégica. Tarragona: Publicacions URV.

Sennett, Richard. 1997. Carne y Piedra. Madrid. Alianza Editorial.

Solà-Morales i Rubió, Manuel. 1985. "Los problemas de la forma urbana: Tarragona, un enfoque telescópico”. Barcelona: Revista UR 1 (1): 44 p.

Solà-Morales i Rubió, Manuel. 2009. "Un camp de coses (els buits de la metròpolis)". Tarragona: Revista AT 19 (1): 3 p.

Zaguirre Fernández, Juan Manuel. 2012. El fenómeno de la industrialización marginal como forma de crecimiento urbano. Libro de Actas del XV CIU Congreso Iberoamericano de Urbanismo. Medellín (Colombia).

Zaguirre Fernández, Juan Manuel. 2015. "Cuatro escenarios para EntrevíasTarragona. ¿Cabría una dinámica dominante?" Alicante: [I2] Innovación e Investigación en Arquitectura y Territorio. 2015, 3(1): 10 p. doi:10.14198/i2.2015.3.01

Zaguirre Fernández, Juan Manuel. 2013. Vías y Entrevías en el horizonte de Tarragona. Exploración, compilación e hipótesis sobre el distrito industrial del Camp de Tarragona. Reus: Tesina-Trabajo Final de Máster. Tutor, J. Parcerisa.

Zaguirre Fernández, Juan Manuel. 2017. Vías y Entrevías. Atributos y compromisos del distrito industrial de Tarragona. Tesis Doctoral. Director: Josep Parcerisa. Barcelona: ETSAB-UPC. 